\title{
Laminin in Lung Development: Effects of Anti-laminin Antibody in Murine Lung Morphogenesis
}

\author{
Lucia Schuger,* Sue O’Shea,† Jill Rheinheimer, $\uparrow$ and James Varani* \\ *Department of Pathology and †Department of Anatomy and Cell Biology, University of Michigan Medical School, Ann Arbor, Michigan 48109
}

Accepted August 29, 1989

\begin{abstract}
The pattern of deposition and the role of laminin, a major glycoprotein constituent of basement membranes, were investigated during lung morphogenesis in the fetal mouse. Lung primordia were removed from Day 13 embryos, right lower lobes were further dissected and placed in filter membrane assemblies. Explants were then cultured at the liquid-air interface for 3 days in the presence of anti-laminin, anti-thrombospondin (another extracellular matrix constituent), preimmune serum, laminin-neutralized anti-laminin, or medium alone. Cultures were monitored by (direct) phase-contrast microscopy, light microscopy, and immunofluorescence. We found that anti-laminin antibodies altered normal lung morphogenesis in a dose-dependent manner. The anti-laminin-treated explants presented a marked inhibition of branching morphogenesis and a distortion of the bronchial tree. A lower rate of growth was also observed in the explants exposed to this antibody. High concentrations of anti-thrombospondin antibodies, normal rabbit serum, or laminin-neutralized anti-laminin antibodies had no effect on lung morphogenesis. These results were not modified by culturing the explants in submersion culture or on Vitrogen 100-coated surfaces. (c) 1990 Academic Press, Inc.
\end{abstract}

\section{INTRODUCTION}

Interaction between epithelium and mesenchyme is required for normal epithelial morphogenesis (Rudnick, 1933; Grobstein, 1967; Spooner, 1974). The extracellular matrix, particularly the basement membrane, which is the interface between epithelium and mesenchyme, is thought to be the site where this interaction occurs and is controlled (Banerjee et al., 1977; Bennett, 1980; Bernfield et al., 1984). Type IV collagen, laminin, heparan sulfate proteoglycan, fibronectin, and entactin are major components of basement membranes (Carlin et al., 1981; Timpl and Martin, 1982); collagens type I, III, and $\mathrm{V}$, elastin, fibronectin, hyaluronate, chondroitin sulfate, and heparan sulfate proteoglycans are components of the mesenchymal matrix (Hay, 1981). The demonstrated functions of these molecules in controlling cellular interactions in vitro and their presence in developing mesenchymal-epithelial organs, including the lung, have lead to speculations as to their role in organogenesis. However, much of the evidence is as yet circumstantial, relying on the fact that their spatial and temporal distributions coincide with key developmental events.

The lack of information about the functional role of extracellular matrix/basement membrane constituents is particularly striking in regard to lung development. Glycosaminoglycans, which appear to have a role in salivary gland branching (Bernfield et al., 1972), have been proposed, as has type IV collagen, to play a role in lung branching development (Bernfield et al., 1984;
Chen and Little, 1987). It has been demonstrated that inhibitors of procollagen synthesis inhibited lung branching morphogenesis (Spooner and Faubion, 1980). Interestly, the studies of Kratochwil et al. (1986) suggest that the presence of type I collagen does not appear to be required for normal development of branching epithelia. Immunohistochemical studies have determined the presence of several other glycoproteins in the developing murine lung ( $\mathrm{Wu}$ et al., 1983; Jaskoll and Slavkin, 1984; O'Shea and Dixit, 1988). However, their roles in lung development remain undetermined.

The present study was undertaken to obtain direct evidence for the involvement of cell-matrix interactions in lung morphogenesis. In this study we used antibodies to two different glycoprotein components of the extracellular matrix in an attempt to interfere with embryonic lung morphogenesis in organ culture. We found that antibodies to one of the matrix molecules, laminin, penetrated the explants, localized at the basement membrane, and produced marked alterations in lung morphogenesis. These results provide direct evidence that interaction of lung epithelial cells with their basement membrane is critical to the process of normal lung development.

\section{MATERIALS AND METIODS}

Isolation and culture of embryonic lungs. CD-1 strain (Charles River) mice were mated and the day of finding a vaginal plug was designated as Day 1 . Pregnant females were sacrificed by cervical dislocation at Day 13 
of gestation. Uteri were removed to sterile phosphatebuffered saline and the lungs were dissected from the embryos with watchmakers forceps, while being viewed through a dissecting microscope. The lower right lobe was further dissected and cultured at the air-medium interface on the upper surface of polycarbonate filter membrane assemblies, $0.4-\mu \mathrm{m}$ pore size (Millipore, Bedford, MA). The culture medium used was minimal essential medium (MEM) (GIBCO, Grand Island, NY) containing $10 \%$ fetal calf serum (Hyclone Lab, Logan, UT), nonessential amino acids, (GIBCO), $0.29 \mathrm{mg} / \mathrm{ml}$ L-glutamine (Irvine Scientific, Santa Ana, CA), 100 $\mathrm{U} / \mathrm{ml}$ penicillin, $100 \mu \mathrm{g} / \mathrm{ml}$ streptomycin, and 0.25 $\mu \mathrm{g} / \mathrm{ml}$ amphotericin B (Irvine Scientific). Cultures were incubated at $37^{\circ} \mathrm{C}$ in $5 \% \mathrm{CO}_{2}$ and fed daily by a complete change of medium. In some experiments the membrane filters were coated with purified collagen, types I and III (Vitrogen 100, Collagen Corp., Palo Alto, CA). In additional experiments the lungs were submerged rather than being placed at the air-medium interface.

Reagents and treatment modalily. Laminin prepared from the Engelbreth-Holm-Swarm tumor by the method of Timpl el al. (1979) was obtained from Collaborative Research (Boston, MA). Upon arrival at the laboratory, each laminin preparation was examined by sodium dodecyl sulfate-polyacrylamide gel electrophoresis (SDS-PAGE) and by enzyme-linked immunosorbent assay (ELISA). Under reducing conditions, only two major protein bands - at 200 and $400 \mathrm{kDa}$ - were seen. The laminin reacted in ELISA with rabbit polyclonal anti-laminin at antibody dilutions between $1: 1$ and 1:10 but did not react with antibodies to type IV collagen, thrombospondin, or fibronectin. The laminin was also examined for biological activity using cell-substrate adhesion as indicator. The laminin was able to induce attachment and spreading in a line of laminin-sensitive murine fibrosarcoma cells.

Rabbit polyclonal antibodies to laminin were prepared by injecting laminin into New Zealand white rabbits. Three subcutaneous injections (at monthly intervals) consisting of $1 \mathrm{mg}$ of laminin in either complement Freund's adjuvent (first injection) or incomplete Freund's adjuvent for the second and third injections. This was followed 1 month later by an intravenous injection of $100 \mu \mathrm{g}$ of laminin. The animals were bled 2-3 weeks after the final injection. The anti-laminin serum was characterized by immunoblotting and by ELISA. Reactivity with both the $400-$ and $200-\mathrm{kDa}$ chains of laminin occurred. When extracts obtained from mouse spleen cells were used as antigen, reactivity was observed only with moieties migrating at 400 and $200 \mathrm{kDa}$ under reducing conditions (Fig. 1). In ELISA, the antilaminin serum reacted with laminin at antibody dilutions between $1: 1$ and $1: 10^{5}$ but did not react with type

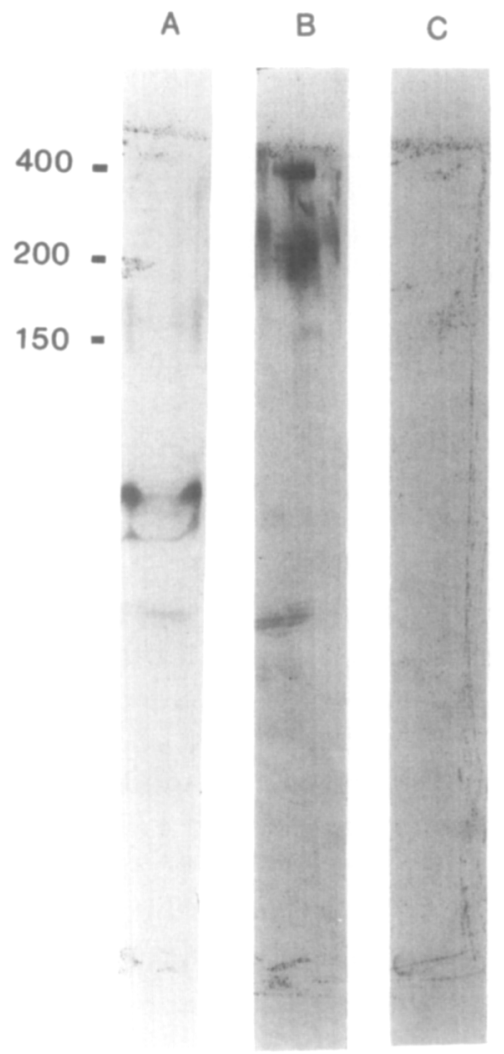

Fig. 1. Analysis by immunoblot of whole spleen cell extracts with anti-laminin. Extracts were prepared from $5 \times 10^{6}$ nonadherent mouse spleen cells and separated by SDS-PAGE in the presence of 2-mercaptoethanol. The separated proteins were transferred to nitrocellulose and probed with a 1:5000 dilution of normal rabbit serum (A) or anti-laminin (B) and ${ }^{125} \mathrm{I}$-protein $\mathrm{A}$. Lane $\mathrm{C}$ shows binding of anti-laminin to buffer alone. Bands were visualized by fluorography after 1 day.

IV collagen, thrombospondin, or fibronectin at any dilution examined. Cross-reactivity against entactin was seen but the antibody titer against this protein was much lower $(50 \%$ end point $=1: 2000$ for entactin vs 1:30,000 for laminin).

Normal rabbit serum and anti-thrombospondin serum were used as controls. The anti-thrombospondin was prepared in New Zealand white rabbits after injection of human platelet thrombospondin. The immunization protocol was similar to that for the preparation of anti-laminin. The thrombospondin was purified from human platelets by gel-filtration and heparin-Sepharose affinity chromatography as described previously (Dixit et al., 1984). The anti-thrombospondin reacted with thrombospondin in ELISA at antibody dilutions between $1: 1$ and $1: 10^{6}$ but did not react with laminin, type IV collagen, or fibronectin at any dilution tested.

Lung explants were allowed to attach to the filter membranes for a period of $4 \mathrm{hr}$ before introducing the 
antibody into the medium. Anti-laminin antibodies and/or anti-thrombospondin antibodies were then added to the culture medium at varying concentrations. Anti-laminin antibodies were used at dilutions of 1:5 (4 experiments, eight lungs), 1:10 (14 experiments), 1:50 (4 experiments), and 1:100 (4 experiments). Anti-thrombospondin antibody was used at concentrations of 50 $\mu \mathrm{g} / \mathrm{ml}$ (3 experiments), $250 \mu \mathrm{g} / \mathrm{ml}$ (3 experiments), and $500 \mu \mathrm{g} / \mathrm{ml}$ (3 experiments). Lung explants were cultured in the presence of the antibody for a period of 3 days. On Days 1-3, organ cultures were observed by phase-contrast microscopy and photographed. During this time, the antibody-containing medium was daily removed and replaced with new medium containing fresh antibodies. Normal rabbit serum was used as a control in place of the antibodies (1:10 dilution in 8 experiments). Other controls consisted of the use of complete media in the presence of neither antibodies nor normal rabbit serum (14 experiments). In an additional 3 experiments the anti-laminin antibodies, at a dilution of 1:10, were preabsorbed with laminin $(100 \mu \mathrm{g} / \mathrm{ml})$ for $30 \mathrm{~min}$ prior to being introduced into the medium.

Light microscopy. Lung explants were fixed in phosphate-buffered formalin, and after fixation, samples were rinsed with $0.1 M$ phosphate buffer and dehydrated through graded alcohols to $100 \%$. Then samples were embedded in glycolmethacrylate (Polysciences, Warrington, PA). Five-micrometer sections were cut, mounted on slides, and stained with hematoxylin-eosin.

Immunohistochemistry. To determine the presence and pattern of deposition of laminin and thrombospondin in normal embryonic lung, lungs removed from Day 13 embryos were immediately frozen in OCT embedding compound (Miles, Naperville, IL) in hexane cooled over an acetone dry-ice slurry. Blocks were stored at $-90^{\circ} \mathrm{C}$ prior to sectioning. Five-micrometer sections were cut using an International cryostat and collected on high molecular weight (g.t. 300,000) poly-L-lysine-coated slides. Sections were air-dried and exposed in a moist chamber to $5 \%$ normal goat serum followed by the primary antibody for $2 \mathrm{hr}$ at room temperature. They were then washed in PBS and exposed to the second antibody (goat anti-rabbit IgG) conjugated to FITC, 1:50 (Cappel, Malvern, PA), for 30 min at room temperature. Sections were washed extensively in PBS and coverslipped with glycerol containing $0.1 \%$ phenylenediamine. Controls were preincubated in preimmune normal rabbit serum or in phosphate-buffered saline in place of the primary antibody.

To determine any possible change in the production and/or distribution of laminin and thrombospondin due to the in vitro conditions, immunostaining was also performed on lung explants maintained in culture for $48 \mathrm{hr}$.
To determine the penetration of anti-laminin or anti-thrombospondin antibodies into the explants, treated and untreated samples were frozen in situ by filling the membrane assembly with $0 \mathrm{CT}$ at $-20^{\circ} \mathrm{C}$. The samples were then processed for immunohistochemistry as above except that the primary antibody (antilaminin or anti-thrombospondin) was omitted during the immunostaining procedure.

\section{RESULTS}

Lung morphogenesis in vitro. At the time of removal from the embryo on Day 13, the lung explants presented a rudimentary branching pattern consisting of five to seven buds. After 3 days in culture, a substantial epithelial branching of the bronchial tree was observed. The five to seven buds present at Time 0 showed a 3- to 4-fold increase in number after $24 \mathrm{hr}$ in culture, a 5- to 6-fold increase after $48 \mathrm{hr}$, and an 8- to 10-fold increase by the third day in culture. A lower rate of branching and growth was observed in vitro as compared to in vivo morphogenesis. An exact correlation between the two, however, was not determined.

Effects of anti-laminin and anti-thrombospondin on lung morphogenesis. Lung explants cultured for 3 days in the presence of anti-thrombospondin showed good branching development even with concentrations as high as $500 \mu \mathrm{g} / \mathrm{ml}$. The presence of normal rabbit serum also did not affect in vitro morphogenesis. Lung explants cultured for 3 days in the presence of anti-laminin antibodies (1:10 dilution) presented severe alterations in morphogenesis. The main findings were a marked epithelial branching inhibition and a distortion of the bronchial tree. A lower growth rate of the explants was also observed (Fig. 2). During the first $48 \mathrm{hr}$ of anti-laminin treatment, the severity of the inhibition in branching morphogenesis was very consistent among different explants. However, by the third day in culture, variation in the degree of branching inhibition could be observed between different explants at the same concentration of antibody. The average number of terminal buds by the third day in culture was 30 (range, 14-35) for the treated explants as compared to 50 (range, 40-75) for the untreated controls and the controls treated with normal rabbit serum. The effects induced by anti-laminin were abrogated by preabsorption of the antibody with laminin for $30 \mathrm{~min}$ prior to introduction into the medium. Anti-laminin-induced changes occurred in all of the experiments when the antibody was used at a dilution of 1:10. Dilutions of 1:50 were effective in three of four experiments. Dilutions of 1:5 were also highly inhibitory of branching morphogenesis and growth in general; a loss of tissue transparence was produced by this high dose. In the presence of high antibody concentrations, occasional explants did not 

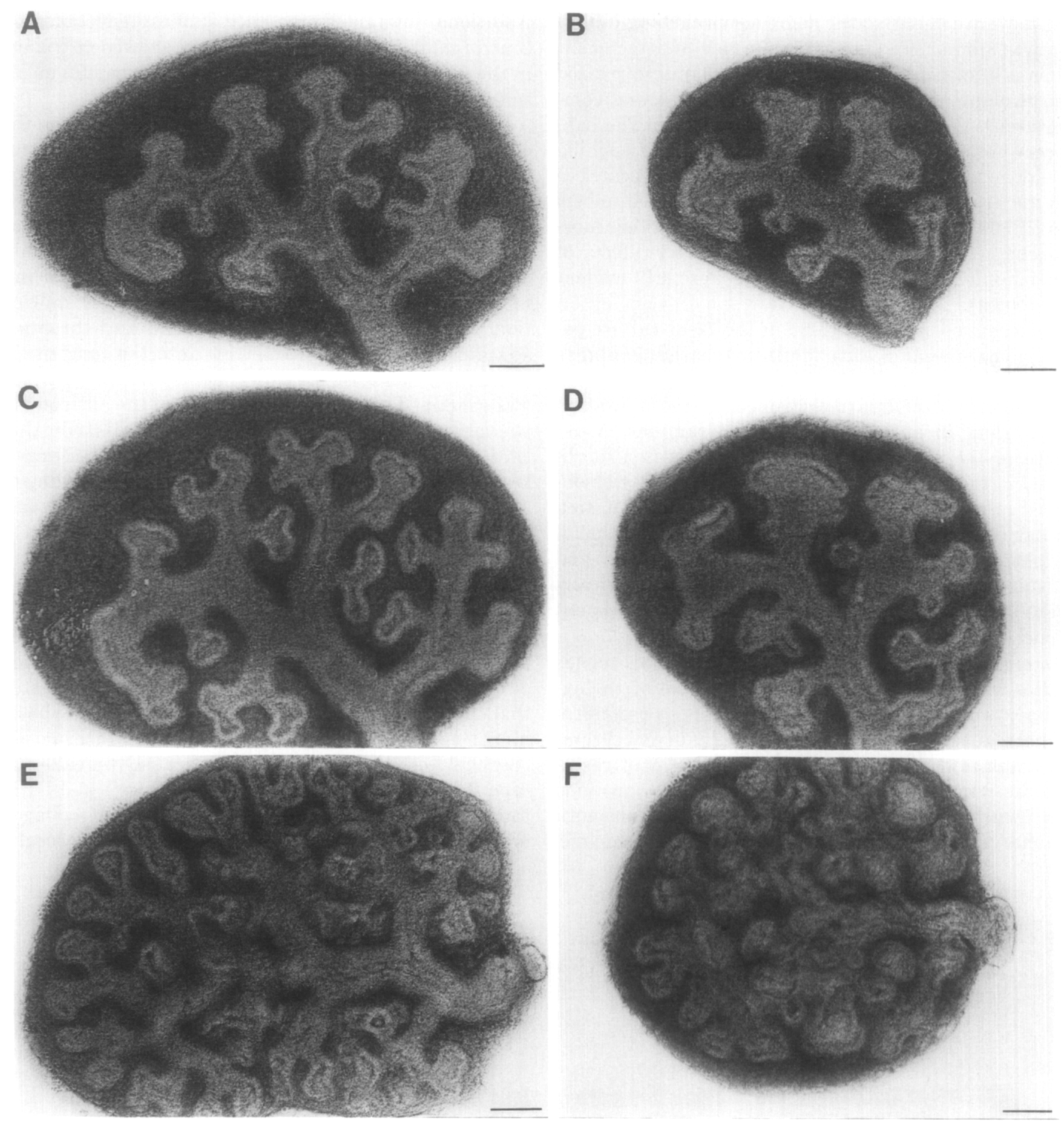

FIG. 2. Lung organ cultures were established on Day 0 and incubated for 3 days in the presence of normal rabbit serum 1:10 dilution (A, $C$, E) or anti-laminin antibodies 1:10 dilution (B, D, F). (A, B) Explant development after $24 \mathrm{hr}$ in culture; the branching morphogenesis inhibition and dilatation of the bronchial tree are already evident in the anti-laminin-treated explant (B). (C, D) Differences observed after 48 hr in culture. (E, F) Explants maintained in normal rabbit serum (E) and anti-laminin (F) for $72 \mathrm{hr}$. The inhibitory effects of anti-laminin on explant growth and particularly on branching morphogenesis are obvious. Scale bars $=100 \mu \mathrm{m}$.

attach to the filter membrane. To ensure attachment of the explants to the membrane surface, some of the filters were coated with collagen (types I and III; Vitrogen 100) before explanting the lung primordia (Chen and Little, 1985). Under these conditions branching morphogenesis occurred to an extent similar to that on uncoated membranes. When the anti-laminin-treated explants cultured on coated and uncoated filter mem- 
branes were compared, the degree of branching inhibition was similar. To determine the role of attachment per se in branching, untreated and anti-laminin-treated lung explants were cultured in submersion and compared with the air-media interface cultures. The submerged culture presented a generally poorer branching development. The buds acquired a saccular appearance and the bronchial tree was dilated. This proved to be a less satisfactory system, therefore, for observing morphogenesis. In spite of this, the inhibitory effects of anti-laminin on lung bud branching were still evident (not shown).

Light microscopy. At the end of the 72-hr culture period, controls and anti-laminin-treated explants showed a total preservation of tissue architecture with maintenance of cellularity. Bronchial tree dilatation was evident in anti-laminin-treated samples. A decrease in the number of mitotic figures was also observed in these explants. Random cell necrosis was observed in all the explants, treated and untreated, to a similar degree. Each group showed the two epithelial morphologies, a proximal tall columnar (bronchial) and a distal (respiratory), that correspond chronologically to the epithelial events occurring in lung development in vivo (Fig. 3).

Immunofluorescence studies. Immunofluorescence studies were undertaken during the course of the experiments for different purposes. First, to reconfirm the normal pattern of deposition of laminin and thrombospondin in embryonic lung at the 13th day of gestation. As was previously reported, both glycoproteins were largely localized in the basement membrane with minimal presence in the epithelium and mesenchyme.

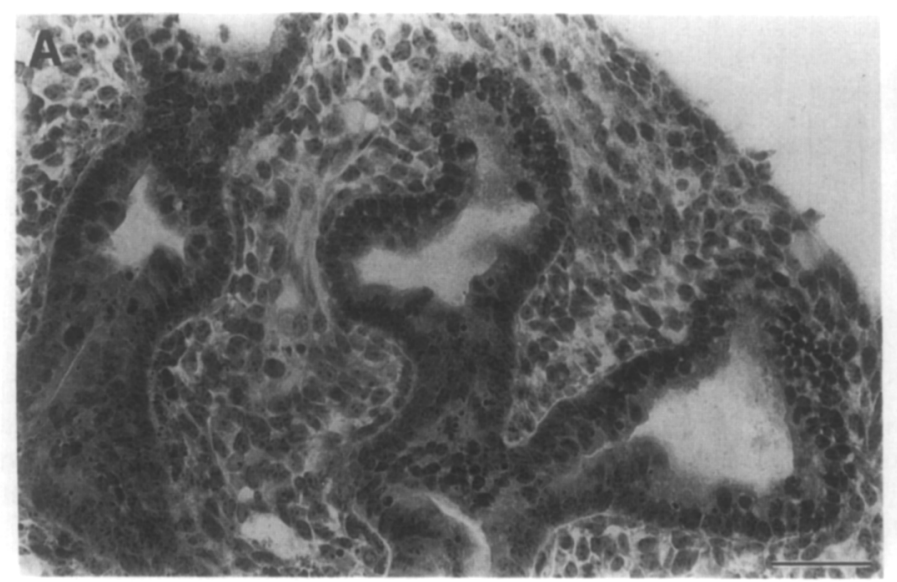

Additional immunofluorescence studies undertaken on control explants after $48 \mathrm{hr}$ in culture showed no change in the distribution of laminin or thrombospondin under in vitro conditions.

Immunohistochemistry was also used to assess the efficiency of the antibodies added to the organ cultures in penetrating the explants. For these experiments, frozen sections from untreated and antibody-treated explants were processed for immunohistochemistry as above except that the primary antibody (anti-laminin or anti-thrombospondin) was omilted during the immunostaining procedure. These experiments showed that both antibodies, anti-laminin and anti-thrombospondin, did effectively penetrate into the lung tissue during the course of the experiments. When the tissue was examined $4 \mathrm{hr}$ after the addition of the antibody to the culture media, there was an even distribution through the parenchyma. When the tissue was examined $18 \mathrm{hr}$ after antibody addition, a specific binding of the antibody to the basement membrane was clearly identified (Fig. 4).

\section{DISCUSSION}

Considerable experimental evidence indicates that normal lung morphogenesis depends on the interaction of epithelium with mesenchyme. It has been suggested that the extracellular matrix and particularly the basement membrane are mediators of this interaction (Bernfield et al., 1984). Since both are rich in collagen, type IV in basement membrane and types I and III in the mesenchyme, it has been proposed that collagen may control epithelial branching behavior. The results

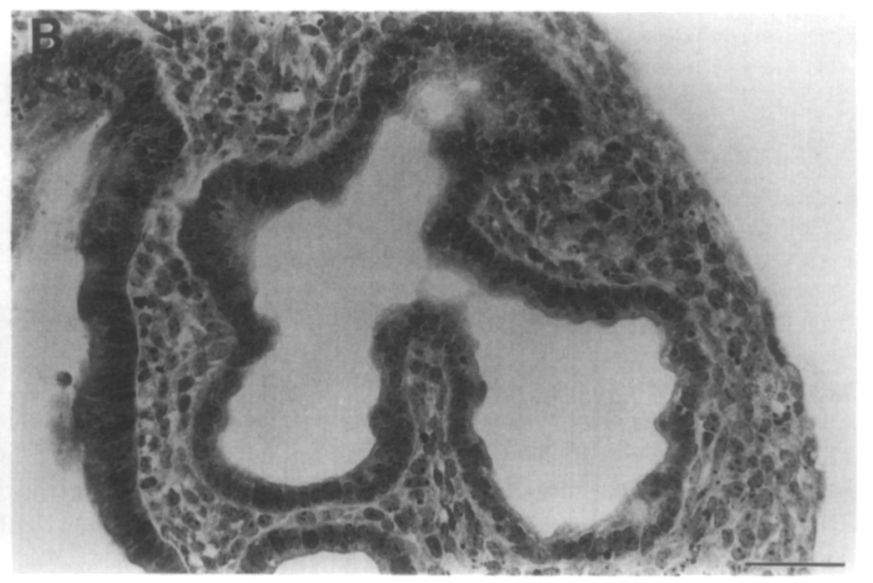

Fig. 3. Longitudinal sections through explants maintained in culture for 3 days in the presence of normal rabbit serum 1:10 dilution (A) or in anti-laminin antibodies 1:10 (B). The tissue presents good viability with full architectural preservation. Note the presence of the two different kinds of epithelium (columnar and cuboidal) expected for this period of development. The control explant (A) shows numerous mitotic figures which are nearly absent in the anti-laminin-treated tissue (B). The bronchial tree in the anti-laminin-treated explant is less highly branched and is dilated compared to the control (A). Scale bars $=50 \mu \mathrm{m}$. 

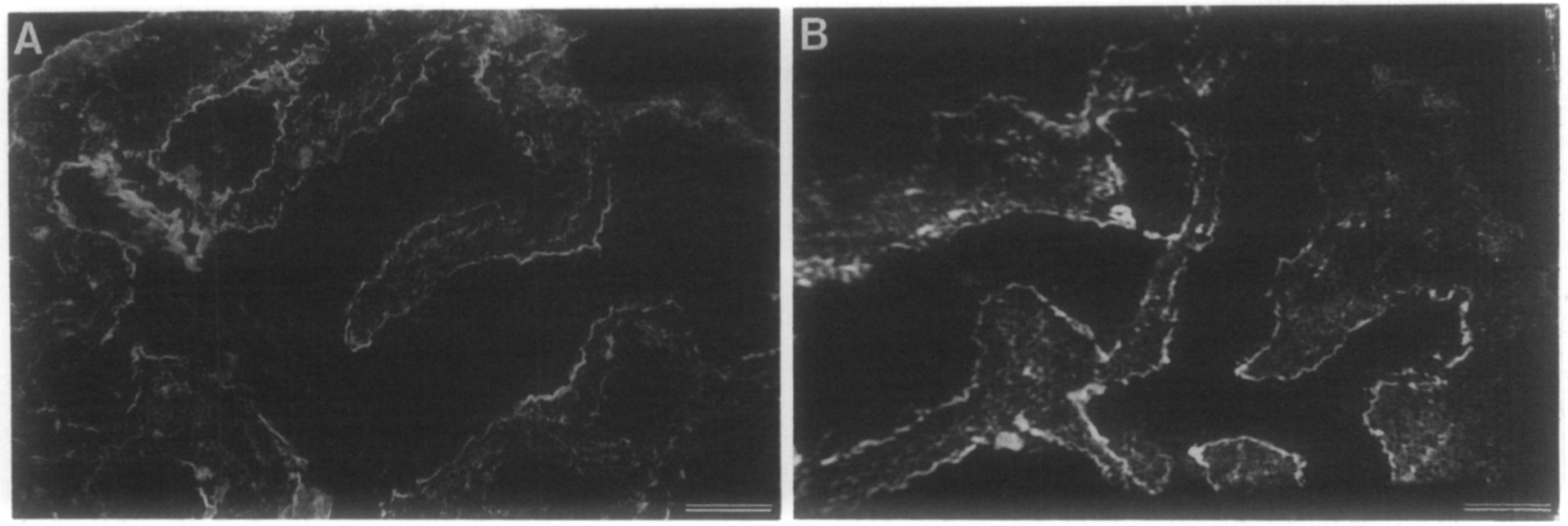

FIG. 4. Immunofluorescence localization of laminin in uncultured lung isolated on the 13th day of development (A) illustrating its deposition in association with the basement membrane of the developing bronchial tree. (B) Penetration of anti-laminin antibody in an explant maintained in vitro for $48 \mathrm{hr}$, sectioned, and exposed to the secondary antibody (goat anti-rabbit IgG) alone. A similar pattern of localization in the epithelial basement membrane was observed. Scale bars $=100 \mu \mathrm{m}$.

obtained using bacterial collagenases (Wessells and Cohen, 1968) or inhibitors of collagen synthesis and secretion (Alecio, 1973; Spooner and Faubion, 1980) support such a possibility. However it has also been demonstrated that development of epithelial-mesenchymal organs, including the lung, can proceed normally, in a collagen I-deficient mouse mutant (Kratochwil et al., 1986). Glycosaminoglycans, which have been extensively studied in the branching of salivary and mammary glands (Bernfield et al., 1972; Saunders and Bernfield, 1988), have also been proposed to be important in lung branching morphogenesis (Bernfield et al., 1984). A similar role for cell adhesion molecules in this process has been suggested by Hirai et al. (1989), who showed that antibodies to two different cell-cell adhesion molecules (E-cadherin and P-cadherin) also interfered with normal morphogenetic events in embryonic lung organ cultures.

If little is known about the roles of collagenous matrix components and glycosaminoglycans in lung morphogenesis, even less is known about other extracellular matrix/basement membrane macromolecules. Glycoproteins such as laminin, fibronectin, and thrombospondin, which are important constituents of lung extracellular matrix, are known from in vitro studies to mediate biological processes such as cell-cell and cellsubstrate adhesion, cell movement, and proliferation (Martin and Timpl, 1987). These biological processes, in turn, are thought to be critical to the normal morphogenetic events that result in the formation of organs such as the lung from collections of epithelial and mesenchymal cells. It might be expected, therefore, that morphogenesis would be altered if cellular interactions with extracellular matrix components such as laminin, fibro- nectin, or thrombospondin could be blocked. Using an embryonic mouse lung organ culture model, in which a highly branched airway forms over a 3-day period from rudimentary tubular structures, the present study provides direct evidence that this is, in fact, what occurs. We were able to show that polyclonal antibodies to laminin penetrated the tissue and localized in basement membranes. In the presence of the antibody, morphogenesis was dramalically altered. Instead of the normal bronchial tree, airways with diffuse, irregular dilatations and enlarged sac-like structures were seen. Antibody dilutions between 1:10 and 1:50 were sufficient to produce these changes (changes were also occasionally seen in the presence of a 1:100 dilution of antibody) and the antibody effects were abrogated when the antibody was first mixed with soluble laminin. The relatively high concentration of antibody required to impair morphogenesis probably reflects the fact that the antibody must penetrate whole, intact tissue to localize at the basement membrane. This is considerably different from a cell culture system in which antibody effectiveness can be based on the ability to interfere with interaction of single cells in suspension with the purified matrix component on the surface of a plastic dish.

How antibody to laminin acts to block morphogenesis is not known and several possibilities must be considered. On the one hand, laminin is known to regulate cell differentiation in vitro, in part by influencing gene expression (Blum et al., 1987). It may be that, in the developing lung, epithelial cell interactions with laminin result in the expression of specific genes that are necessary for normal development of the bronchial tree.

Alternatively, the presence of the antibody may inhibit normal morphogenesis as a more global conse- 
quence of interfering with cell-basement membrane interaction. Proliferation of epithelial cells is an anchorage-dependent phenomenon and growth could be slowed as a result of the antibody interfering with cell attachment to the basement membrane. Possibly, antibodies to other basement membrane constituents would produce similar effects. In this regard it should be noted that the various anti-laminin preparations used in this study show some cross-reactivity with entactin, at least by ELISA. Since entactin is intimately associated with laminin and, like laminin, localized in basement membranes, it is possible that anti-entactin antibodies in the antiserum may contribute to altered morphogenesis. It is also possible that antibodies bound to either protein may sterically hinder interactions with the other. Although experiments in which normal morphogenesis occurred when anti-laminin antibodies were preabsorbed with laminin strongly suggest that the effects may be due to interference with cell-laminin interactions, additional experiments must be done before these possibilities can be distinguished.

Eventually, the presence of the antibody may block domains of laminin with growth-factor activity (Panayotou et al., 1989); the dramatic decrease in mitotic figures observed in the treated explants supports this possibility.

The present studies provide direct evidence that antibodies which penetrate primordial lung organ buds in culture and bind to basement membranes interfere with normal organ development over a 3-day period. Future studies will be directed toward understanding the mechanisms by which the antibodies interfere with morphogenesis. It is hoped that this, in turn, will lead to an understanding of morphogenesis at the cellular level.

This research was supported by Grants IM-432 and PDT-324 from the ACS and Grant HD-23867 from the NIH.

\section{REFERENCES}

ALECIO, T. (1973). Effect of a proline analogue, azetidine 2-carboxylic acid, on the morphogenesis in vitro of mouse embryonic lung. $J$. Embryol. Exp. Morphol. 29, 493-451.

BanerJee, S. D., R. H. Cohn, and M. Bernfield. (1977). Basal lamina of embryonic salivary epithelium: Production by the epithelium and role in maintaining lobular morphology. J. Cell Biol 73, 445-463.

BennetT, D. C. (1980). Morphogenesis of branching tubules in cultures of cloned mammary epithelial cells. Nature (London) 285, 657-659.

Bernfield, M. R., BanerJee, S. D., and Cohn, R. H. (1972). Dependence of salivary epithelial morphology and branching morphogenesis upon acid mucopolysaccharide-protein (protcoglycan) at the epithelial surface. J. Cell Biol. 52, 674-689.

Bernfield, M., BanerJee, S. D., Koda, J. E., and RAPraeger, A. C. (1984). Remodeling of the basement membrane as a mechanism of morphogenetic tissue interaction. In "The Role of Extracellular Matrix in Development" (R. L. Trestad, Ed.), pp. 545-572. A. R. Liss, New York.

BLUM, J., ZIEGLER, M. E, and WICHA, M. S. (1987). Regulation of rat mammary gene expression by extracellular matrix components. Exp. Cell Res. 173, 322-340.

CARLin, B., JAFFe, R., Bender, B., and ChUnG, A. E. (1981). Entactin, a novel basal lamina-associated sulfated glycoprotein. J. Biol. Chem. 256, 5209-5214.

Chen, J. M., and LiTTLe, C. D. (1985). Cells that emerge from embryonic explants produce fibers of type IV collagen. J. Cell Biol. 101, 1175-1181.

Chen, J. M., and LIITLE, C. D. (1987). Cellular events associated with lung branching morphogenesis including the deposition of collagen type IV. Dev. Biol. 120, 311-321.

Dixit, V. M., Grant, G. A., SANToro, S. A., and Frazier, W. A. (1984). Isolation and characterization of a heparin-binding domain from the amino terminus of platelet thrombospondin. J. Biol. Chem. 259, 10,100-10,105.

GrobsteIn, C. (1967). Mechanism of organogenetic tissue interaction. Natl. Cancer Inst. Monogr. 26, 279-299.

HAY, E. (1981). Collagen in embryonic development. In "Cell Biology of the Extracellular Matrix" (E. Hay, Ed.), pp. 379-409. Plenum, New York.

Hirai, Y., Nose, A., Kobayashi, S., and Takeichi, M. (1989). Expression and role of $\mathrm{E}$ - and $\mathrm{P}$-cadherin adhesion molecules in embryonic histogenesis. Development 105, 263-270.

JASKOLL, T. F., and SLAVKIN, C. (1984). Ultrastructural and immunofluorescence studies of basal-lamina alterations during mouse-lung morphogenesis. Differentiation 28, 36-48.

Kratochwil, K., Dziadek, M., Lohler, J., Harbers, K., and JAENISCH, R. (1986). Normal epithelial branching morphogenesis in the absence of collagen I. Dev. Biol. 117, 596-606.

MarTin, G. R., and TIMPL, R. (1987). Laminin and other basement membrane components. Annu. Rev. Cell. Biol. 3, 57-85.

O'SheA, K. S., and DrXIT, V. M. (1988). Unique distribution of the extracellular matrix component thrombospondin in the developing mouse embryo. J. Cell Biol. 107, 2737-2748.

Panayotou, G., End, P., Aumallley, M., Timpl, R., and Engel, J. (1989). Domains of laminin with growth-factor activity. Cell 56, 93-101.

RuDNick, D. (1933). Developmental capacities of the chick lung in chorioallantoic grafts. J. Exp. Zool. 66, 125-154.

SAUnders, S., and BERnfield, M. (1988). Cell surface proteoglycan binds mouse mammary epithelial cells to fibronectin and behaves as a receptor to interstitial matrix. $J$. Cell Biol. 106, 423-430.

SPOONER, B. S. (1974). Murphogenesis of vertebrate organs. In "Concepts of Development" (J. Lash and J. R. Whittaker, Eds.), pp. 213-240. Sinauer, Stamford, CT.

SpoONER, B. S., and FaUbion, J. M. (1980). Collagen involvement in branching morphogenesis of embryonic lung and salivary gland. Dev. Biol. 77, 84-102.

Timpl, R., and Martin, G. R. (1982). Components of basement membranes. In "Immunochemistry of the Extracellular Matrix" (H. Furthermayr, Ed.), Vol. 2, pp. 119-150. CRC Press, Boca Raton, FL.

Timpl, R., RoHde, M., GeHron-Robey, P., REnNaRd, S. T., FoIdart, J. M., and MARTIN, G. R. (1979). Laminin-A glycoprotein from basement membranes. J. Biol. Chem. 254, 9933-9937.

WESSELLS, N. K., and CoHEN, J. H. (1968). Effects of collagenase on developing epithelia in vitro: Lung, ureteric bud, and pancreas. Dev. Biol. 18, 294-309.

Wu, T. C., WaN, Y. J., Chung, A. E., and Damjanov, I. (1983). Immunohistochemical localization of entactin and laminin in mouse embryos and fetuses. Dev. Biol. 100, 496-505. 